\title{
Medical Plant and Human Health
}

\author{
Ahmed Morsy Ahmed \\ Faculty of Agriculture, \\ Ain shams University, \\ Egypt
}

\section{Introduction}

\subsection{Primary healthcare and traditional medicine}

Primary Health Care (PHC) is the key to the development of a national health policy based on practical, scientifically sound and socially acceptable methods and technology made universally acceptable to individuals and families in the community and through their full participation and at a cost that the community and the country can afford, in order to maintain, at every stage of their development, in the spirit of self-reliance and self determination. It is the first level of contact for the individual, family and the community within the national health care system, bringing health care as close as possible to where people live and work and thus constitutes the first element of a continuing health care process (WHO, 1978a). A health system, based on primary health care was adopted as the means of achieving the goal of health for all. Most developing countries of the world, for which the scheme was designed, have failed to seriously implement it up till this moment.

Traditional Medicine is defined by the World Health Organization (WHO, 1978a) as the sum total of knowledge or practices whether explicable or inexplicable, used in diagnosing, preventing or eliminating a physical, mental or social disease which may rely exclusively on past experience or observations handed down from generation to generation, verbally or in writing. It also comprises therapeutic practices that have been in existence often for hundreds of years before the development of modern scientific medicine and are still in use today without any documented evidence of adverse effects. The explicable form of Traditional Medicine can be described as the simplified, scientific and the direct application of plant, animal or mineral materials for healing purposes and which can be investigated, rationalized and explained scientifically.

\subsection{Herbal medicine}

The use of Salix alba, the willow plant (containing the salicylates) for fever and pains which led to the discovery of aspirin, would belong to this form of Traditional Medicine. Herbal medicines, which squarely belong to this form, are regarded by the World Health Organization, as finished and labeled medicinal products that contain, as active ingredients, aerial or underground parts of identified and proven plant materials, or combination thereof, whether in crude form or as plant preparations. They also include plant juices, gums, fatty oils, essential oils etc (WHO, 1978a). There are several other official modern drugs today, which were originally developed like aspirin through traditional medicine e.g. 
morphine, digoxin, quinine, ergometrine, reserpine, atropine, etc and all of which are currently being used by orthodox medicine in modern hospitals all over the world.

The inexplicable form of Traditional Medicine on the other hand, is the spiritual, supernatural, magical, occultic, mystical, or metaphysical form that cannot be easily investigated, rationalized or explained scientifically e.g. the use of incantations for healing purposes or oracular consultation in diagnosis and treatment of diseases. The explanation is beyond the ordinary scientific human intelligence or intellectual comprehension. Plants are reputed in the indigenous systems of medicine for the treatment of various diseases (Arise et al., 2009). Phyto-chemicals isolated from plant sources are used for the prevention and treatment of several medical problems including diabetes mellitus (Waltner- Law et al., 2002). There are more than 800 plant species showing a hypoglycemic activity. The World Health Organization (1980) has also recommended the evaluation of the effectiveness of plants in conditions where safe modern drugs are lacked.

\section{Major groups of antimicrobial compounds from plants}

Plants have an almost limitless ability to synthesize aromatic substances, most of which are phenols or their oxygen-substituted derivatives (Geissman 1963). Most are secondary metaboltes, of which at least 12,000 have been isolated, a number estimated to be less than $10 \%$ of the total (Schultes 178). In many cases, these substances serve as plant defense mechanisms against predation by microorganisms, insects, and herbivores. Some, such as terpenoids, give plants their odors; others (quinones and tannins) are responsible for plant pigment. Many compounds are responsible for plant flavor (e.g., the terpenoid capsaicin from chili peppers), and some of the same herbs and spices used by humans to season food yield useful medicinal compounds.

Simple phenols and phenolic acids, The mechanisms thought to be responsible for phenolic toxicity to microorganisms include enzyme inhibition by the oxidized compounds, possibly through reaction with sulfhydryl groups or through more nonspecific interactions with the proteins (Mason and Wasserman 1987).Phenolic compounds possessing a $C_{3}$ side chain at a lower level of oxidation and containing no oxygen are classified as essential oils and often cited as antimicrobial as well. Eugenol is a well-characterized representative found in clove oil. Eugenol is considered bacteriostatic against both fungi (Duke 1985) and bacteria (Thomson 1978). Quinones are aromatic rings with two ketone substitutions. They are ubiquitous in nature and are characteristically highly reactive. Probable targets in the microbial cell are surface-exposed adhesins, cell wall polypeptides, and membrane-bound enzymes. Quinones may also render substrates unavailable to the microorganism. As with all plant-derived antimicrobials, the possible toxic effects of quinones must be thoroughly examined.Kazmi et al.

Flavones, flavonoids, and flavonols activity is probably due to their ability to complex with extracellular and soluble proteins and to complex with bacterial cell walls, as described above for quinones. More lipophilic flavonoids may also disrupt microbial membranes (Tsuchiya 1996). Tannins are found in almost every plant part: bark, wood, leaves, fruits, and roots (Scalbert 1991). This group of compounds has received a great deal of attention in recent years, since it was suggested that the consumption of tannin-containing beverages, especially green teas and red wines, can cure or prevent a variety of ills (Serafini et al 1994) their mode of antimicrobial action, may be related to their ability to inactivate microbial adhesins, enzymes, cell envelope transport proteins, etc. They also complex with 
polysaccharide (Ya et al 1988). The antimicrobial significance of this particular activity has not been explored. There is also evidence for direct inactivation of microorganisms: low tannin concentrations modify the morphology of germ tubes of Crinipellis perniciosa (Brownlee et al 1990). Tannins in plants inhibit insect growth (Schultz 1988) and disrupt digestive events in ruminal animals (Butler 1988).

Terpenoids and Essential Oils are secondary metabolites that are highly enriched in compounds based on an isoprene structure. They are called terpenes, their general chemical structure is $\mathrm{C}_{10} \mathrm{H}_{16}$, and they occur as diterpenes, triterpenes, and tetraterpenes $\left(\mathrm{C}_{20}, \mathrm{C}_{30}\right.$, and $\left.C_{40}\right)$, as well as hemiterpenes $\left(C_{5}\right)$ and sesquiterpenes $\left(C_{15}\right)$. When the compounds contain additional elements, usually oxygen, they are termed terpenoids. The mechanism of action of terpenes is not fully understood but is speculated to involve membrane disruption by the lipophilic compounds. Accordingly, Mendoza et al. (Mendoza et al 1997) found that increasing the hydrophilicity of kaurene diterpenoids by addition of a methyl group drastically reduced their antimicrobial activity.

Alkaloids are Heterocyclic nitrogen compounds. Alkaloids have been found to have microbiocidal effects (including against Giardia and Entamoeba species Ghosha et al 1996, the major antidiarrheal effect is probably due to their effects on transit time in the small intestine. Berberine is an important representative of the alkaloid group. It is potentially effective against trypanosomes (Freiburghaus et al 1996) and plasmodia Omulokoli et al 1997). The mechanism of action of highly aromatic planar quaternary alkaloids such as berberine and harmane (Hopp 1976) is attributed to their ability to intercalate with DNA (Phillipson and O'Neill 1987). The mechanism of lectins and polypeptides may be back to the formation of ion channels in the microbial membrane (Terras et al. 1993) or competitive inhibition of adhesion of microbial proteins to host polysaccharide receptors (Sharon and Ofek 1986). Recent interest has been focused mostly on studying anti-HIV peptides and lectins, but the inhibition of bacteria and fungi by these macromolecules, such as that from the herbaceous Amaranthus, has long been known (De Bolle et al 1996).

\subsection{Ethnobotanical study}

Out of around 1076 species recorded so far from Semifinal Biosphere Reserve, more than 200 species are attributed with medicinal uses (Rout, 2004). This system of using herbs and different biological active ingredients in treating various diseases had become a part of their culture till recent years. Entrance of market economy gave rise to exploitation of natural resources and thereby depleting our resources base. The most affected part in this process was medicinal plants, which is most sensitive and delicate in the environment of forest. These medicinal plants gain further importance in the region where modern medical health facilities are either not available or not easily accessible. Although our ancient sages through hit and trial method developed herbal medicines, the reported uses of plant species do not certify efficacy.

The present preliminary report on the uses of some plant species need to pharmacologically screened, chemically analyzed and tested for bioactive activities. Pharmacological screening of plant extracts provides insight to both their therapeutic and toxic properties and helps in eliminating the medicinal plants or practices that may be harmful. The plant parts used for medical preparation were bark, flowers, rhizomes, roots, leaves, seeds, gum and whole plants. In some cases the whole plant including roots was utilized. The use of plant derived products containing high of dietary fiber and complex polysaccharide for the management of diabetes have been proposed (Jenkins et al., 1976). Natural products especially of plant 
origin have been found to be potential sources of novel molecules for the treatment of diabetes (Farnsworth, 1994; Marles and Farnsworth, 1995).

Considering the rate at which the vegetation is getting depleted in this part of the world, therefore it is needed to document the precious knowledge of these plants and to search for more plants with antidiabetic potential. The search for anti-diabetic agents has been focused on plants because of their ready availability, effectiveness, affordability and probably due to low side effects (Marles and Farnsworth, 1995). Ethnobotanical study has been the method often used to search for locally important plant species with low side effects especially for the discovery of crude drugs (Farnsworth, 1994). The present study therefore is a documentation of plants and plant parts used for the management of diabetes mellitus by traditional healers of the area (Table 1).

\begin{tabular}{|c|l|}
\hline Diagnosis methods & Respondent (\%) \\
\hline Signs and symptoms & 100 \\
Loss of body weight & 100 \\
Body weakness & 100 \\
Excessive urination & 100 \\
Presence of sugar in urine & 53 \\
Excessive thirsty & \\
\hline Duration of treatment & 40 \\
Short duration & 60 \\
\hline Long duration & \\
\hline Efficacy of plant treatment on patients & 100 \\
\hline Disappearance of sugar in urine 100 & 100 \\
Reduction in body weakness 100 & 100 \\
Normal body weight 100 & 100 \\
\hline Reduction in frequency of urination & \\
\hline Yes & 100 \\
No & 0 \\
\hline Traditional healers claim of no adverse effect after treatment & 80 \\
\hline Yes & 20 \\
\hline No &
\end{tabular}

Table 1. Diagnosis methods of diabetes mellitus by the herbalists using herbal preparation

\subsection{Diabetes mellitus}

\subsubsection{What is Diabetes?}

The word "diabetes" (a Greek word that means "to pass through") was first used by Aretaeous of Capadocia in the $2^{\text {nd }}$ century AD to describe a condition that is characterized by excess of sugar in blood and urine, hunger and thirst (MacFrlance et al.,1997) and the adjective "mellitus" (a latin-greek word that means "honey") was introduced by the English physician John Rollo so as to distinguish the conditions from other polyuric diseases, in which glycosuria does not occur (Rollo 1797). People suffering from diabetes are not able to produce or properly use insulin in the body and therefore chronic hyperglycemia occurs. In addition, the diabetic individual is prone to late onset complications (Fujisawa et al., 2004), such as retinopathy, neuropathy and vascular diseases, that are largely responsible for the morbidity and mortality observed in diabetic patients. 
Diabetes mellitus is a chronic metabolic disorder characterized by widespread complications. It is the world's largest endocrine disease associated with increased morbidity and mortality rates (Ghosh and Surawanshi, 2001). The chronic hyperglycemia of diabetes is associated with long-term damage, dysfunction and failure of various organs (Lyra et al., 2006). Liver involvement is one of the leading causes of death in diabetes mellitus. The mortality rate from the hepatic affection is greater than that from the cardiovascular complications. The spectrum of liver implication in diabetes ranges from non-alcoholic fatty liver disease to cirrhosis and eventually hepato-cellular carcinoma (Keith et al., 2004). Liver, an insulin-dependent organ, plays a pivotal role in glucose and lipid homeostasis. It participates in the uptake, oxidation and metabolic conversion of free fatty acids and in the synthesis of cholesterol, phospholipids and triglycerides.

Several mechanisms are implicated in the pathogenesis of the functional and morphological alterations of the liver of diabetic patients (Brixova, 1981; Moller, 2001). There are two main types of diabetes, namely type I and type II (World Health Organization. Definition, Diagnosis and Classification of Diabetes mellitus and its omplications. Part 1: Diagnosis and Classification of Diabetes Mellitus (Department of Non communicable Disease Surveillance, Geneva, 1999)). Type I Diabetes, that is called insulin-dependent diabetes mellitus (IDDM) or juvenile onset diabetes develops when the body's immune system destroys pancreatic $\beta$ ells, the only cells in the body that produce the hormone insulin that regulates blood glucose. This type of diabetes usually strikes children and adults and the need for insulin administration is determinant for survival. Type I diabetes accounts for $5 \%$ to $10 \%$ of all diagnosed cases of diabetes and the risk factors may be autoimmune, genetic, or environmental.

On the other hand, type II diabetes, also called non-insulin-dependent diabetes mellitus (NIDDM) or adult-onset diabetes, accounts for about $90 \%$ to $95 \%$ of all diagnosed cases of diabetes. It usually begins as insulin resistance, a disorder in which the cells do not use insulin properly and as the need for insulin rises; the pancreas gradually loses its ability to produce it. This type of diabetes is associated with older age, obesity, family history of diabetes, history of gestational diabetes, impaired glucose metabolism, physical inactivity, and race/ethnicity. It must be noted thought that in the last decay type II diabetes in children and adolescents is being diagnosed more frequently (Fagot-Gampagna \& Narayan 2001). In the case of the IDDM, insulin is of crucial importance for the survival of the patients. On the other hand, in the case of NIDDM the treatment includes medicines, diets and physical training.

\subsubsection{Ant diabetic medicines: Important medicinal plants used in diabetes treatment; important active}

\subsubsection{Compounds involved in diabetes mellitus treatment}

Many kinds of antidiabetic medicines have been developed for the patients and most of them are chemical or biochemical agents aiming at controlling or/and lowering blood glucose to a normal level. Despite the impressive advances in health sciences and medical care, there are many patients who are using alternative therapies alone or complementary to the prescribed medication. Traditional plant remedies or herbal formulations exist from ancient times and are still widely used, despite all the controversy concerning their efficacy and safety (Huxtable 1990; Fugh-Berman 2000), to treat hypoglycemic and hyperglycemic conditions all over the world. 
It must be noted that many ethno-botanical surveys on medicinal plants used by the local population have been performed in different parts of the world and there is a considerable number of plants described as antidiabetic. In addition a variety of compounds have been isolated (alkaloids, glycosides, terpenes, flavonoids, etc) but further studies need to be done so as these 'leads' to develop into clinically useful medicines. To date, met form in (a biguanide) is the only drug approved for treatment of type II diabetes mellitus. It is a derivative of an active natural product, galegine, isolated from the plant Galega officinalis L. (Witters 2001). The followinf table illustrate some of these plant involved in diabetes mellitus treatment

\begin{tabular}{|c|c|c|c|c|}
\hline $\begin{array}{c}\text { Scientific } \\
\text { name }\end{array}$ & Family & $\begin{array}{c}\text { Active } \\
\text { constituent }\end{array}$ & Part Used & Folk Medical Uses \\
\hline $\begin{array}{l}\text { Abelmoschus } \\
\text { moschatus }\end{array}$ & Malvaceae & myricetin & aerial part & $\begin{array}{l}\text { hypoglycemic action decrease } \\
\text { the plasma glucose } \\
\text { concentrations myricetin has an } \\
\text { ability to enhance glucose } \\
\text { utilization to lower plasma } \\
\text { glucose in diabetic rats with } \\
\text { deficient insulin } \\
\text { levels. }\end{array}$ \\
\hline $\begin{array}{l}\text { Azadirachta } \\
\text { indica }\end{array}$ & Meliaceae & & $\begin{array}{c}\text { dried } \\
\text { powder of } \\
\text { root and } \\
\text { leaves }\end{array}$ & $\begin{array}{l}\text { caused significant lowering of } \\
\text { blood sugar and reduction in } \\
\text { serum lipids }\end{array}$ \\
\hline $\begin{array}{l}\text { Cornus } \\
\text { macrophylla }\end{array}$ & Cornaceae & & & $\begin{array}{l}\text { prevent and treat diabetic } \\
\text { complications }\end{array}$ \\
\hline $\begin{array}{l}\text { Achyranthes } \\
\text { aspera } L \text {. }\end{array}$ & Amaranthaceae & & $\begin{array}{c}\text { powdered } \\
\text { whole } \\
\text { plant }\end{array}$ & hypoglycemic effect \\
\hline $\begin{array}{l}\text { Achyrocline } \\
\text { satureioides }\end{array}$ & Asteraceae & achyrofuran & $\begin{array}{c}\text { powdered } \\
\text { whole } \\
\text { plant }\end{array}$ & lowered blood glucose levels \\
\hline $\begin{array}{l}\text { Acosmium } \\
\text { panamense }\end{array}$ & Fabaceae & & $\begin{array}{c}\text { water and } \\
\text { butanolic } \\
\text { extracts }\end{array}$ & $\begin{array}{l}\text { lowered the plasma glucose } \\
\text { levels }\end{array}$ \\
\hline $\begin{array}{c}\text { Aegle } \\
\text { marmelose }\end{array}$ & Rutaceae & & leaf extract & $\begin{array}{l}\text { similar hypoglycemic activity to } \\
\text { that of insulin treatment } \\
\text { Treatment with the leaf extract } \\
\text { showed improved functional } \\
\text { state of pancreatic } \beta \text { cells. The } \\
\text { results indicate the potential } \\
\text { hypoglycemic effect of the leaf } \\
\text { extract, possibly involved in } \\
\text { processes for the regeneration of } \\
\text { damaged pancreas. }\end{array}$ \\
\hline
\end{tabular}

Table 2. Important medicinal Plants used in Diabetes treatment. 


\begin{tabular}{|c|c|c|c|c|}
\hline $\begin{array}{c}\text { Scientific } \\
\text { name }\end{array}$ & Family & $\begin{array}{c}\text { Active } \\
\text { constituent }\end{array}$ & Part Used & Folk Medical Uses \\
\hline $\begin{array}{l}\text { Allium } \\
\text { sativum L }\end{array}$ & (Liliaceae & allicine & & $\begin{array}{l}\text { decreased the concentration of } \\
\text { serum lipids, blood glucose and } \\
\text { activities of serum enzymes like } \\
\text { alkaline phosphatase, acid } \\
\text { phosphatase and lactate } \\
\text { dehydrogenase and liver } \\
\text { G6Pase }\end{array}$ \\
\hline \multirow[t]{2}{*}{$\begin{array}{l}\text { Allium } \\
\text { cepa L. }\end{array}$} & (Liliaceae & $\begin{array}{l}\text { S-methyl } \\
\text { cysteine } \\
\text { sulfoxide }\end{array}$ & & $\begin{array}{l}\text { lowered the levels of } \\
\text { malondialdehyde, } \\
\text { hydroperoxide and conjugated } \\
\text { dienes in tissues exhibiting } \\
\text { antioxidant effect }\end{array}$ \\
\hline & & (SMCS) & & $\begin{array}{l}\text { on lipid peroxidation in } \\
\text { experimental diabetes The } \\
\text { hypoglycemic and } \\
\text { hypolipidaemic actions of } \\
\text { Allium cepa were associated } \\
\text { with antioxidant activity, since } \\
\text { onion decreased SOD activities } \\
\text { while no increased lipid } \\
\text { hydroperoxide and lipoperoxide } \\
\text { concentrations were observed in } \\
\text { diabetic rats treated with Allium } \\
\text { cepa }\end{array}$ \\
\hline Aloe vera & (Liliaceae & & $\begin{array}{l}\text { leaf pulp } \\
\text { and gel } \\
\text { extracts }\end{array}$ & $\begin{array}{l}\text { contain a hypoglycemic agent } \\
\text { which lowers the blood glucose } \\
\text { levels showed hypoglycemic } \\
\text { activity on type I and II diabetic } \\
\text { maintained the glucose } \\
\text { homeostasis by controlling the } \\
\text { carbohydrate metabolizing } \\
\text { enzymes } \\
\text { (Rajasekaran et al., 2004). }\end{array}$ \\
\hline $\begin{array}{c}\text { Aloe } \\
\text { barbadensis }\end{array}$ & Liliaceae & & leaves & $\begin{array}{l}\text { the bitter principle may be } \\
\text { mediated through stimulating } \\
\text { synthesis and/or release of } \\
\text { insulin from the } \beta \text { cells of } \\
\text { Langerhans }\end{array}$ \\
\hline $\begin{array}{l}\text { Andrographis } \\
\text { paniculata }\end{array}$ & Acanthaceae & andrographolide & $\begin{array}{c}\text { crude } \\
\text { ethanolic } \\
\text { extract }\end{array}$ & $\begin{array}{l}\text { antidiabetic effect may be } \\
\text { attributed, at least in part, to } \\
\text { increased glucose metabolism } \\
\text { andrographolide can increase } \\
\text { the glucose utilization to lower } \\
\text { plasma glucose in diabetic rats } \\
\text { lacking insulin. }\end{array}$ \\
\hline
\end{tabular}

Table 2. Important medicinal Plants used in Diabetes treatment. (continuation) 


\begin{tabular}{|c|c|c|c|c|}
\hline $\begin{array}{l}\text { Scientific } \\
\text { name }\end{array}$ & Family & $\begin{array}{c}\text { Active } \\
\text { constituent }\end{array}$ & Part Used & Folk Medical Uses \\
\hline $\begin{array}{l}\text { Angylocalyx } \\
\text { pynaertii }\end{array}$ & Leguminosae & $\begin{array}{l}\text { sugar-mimic } \\
\text { alkaloids }\end{array}$ & $\begin{array}{c}\text { pod } \\
\text { extract }\end{array}$ & $\begin{array}{l}\text { specific inhibitors of alpha-L } \\
\text { fucosidase with no significant } \\
\text { inhibitory activity towards other } \\
\text { glycosidases }\end{array}$ \\
\hline Areca catechu & & arecolin & & $\begin{array}{l}\text { Arecoline have hypoglycemic } \\
\text { activity in animal model of } \\
\text { diabetes upon s.c. } \\
\text { administration }\end{array}$ \\
\hline $\begin{array}{l}\text { Averrhoa } \\
\text { bilimbi L. }\end{array}$ & Oxalidaceae & & $\begin{array}{l}\text { ethanolic } \\
\text { extract }\end{array}$ & $\begin{array}{l}\text { ethanolic extract has } \\
\text { hypoglycemic, } \\
\text { hypotriglyceridemic, anti-lipid } \\
\text { peroxidative and anti- } \\
\text { atherogenic properties in STZ- } \\
\text { diabetic rats. }\end{array}$ \\
\hline $\begin{array}{l}\text { Bauhinia } \\
\text { forficata } L \text {. }\end{array}$ & Leguminosae & kaempfer itr in & $\begin{array}{c}\text { n-butanol } \\
\text { extract }\end{array}$ & $\begin{array}{l}\text { blood glucose-lowering effect in } \\
\text { normal and diabetic rats }\end{array}$ \\
\hline $\begin{array}{l}\text { Beta vulgaris } L \text {. } \\
\text { var. cicla }\end{array}$ & Chenopodiaceae & $\begin{array}{l}\text { Betavulgaroside } \\
\text { I, II, III and IV }\end{array}$ & roots & $\begin{array}{l}\text { hypoglycemic effects that was } \\
\text { demonstrated by a per os } \\
\text { glucose tolerance test in rats } \\
\text { after their per os administration }\end{array}$ \\
\hline Bidens pilosa & Asteraceae & $\begin{array}{l}\text { 3-beta-D- } \\
\text { glucopyranosylo } \\
\text { xy-1-hydroxy- } \\
\text { 6(E)-tetradecene- } \\
\text { 8,10,12-triyne }\end{array}$ & $\begin{array}{l}\text { Butanol } \\
\text { extract of } \\
\text { whole } \\
\text { plant }\end{array}$ & $\begin{array}{l}\text { inhibited the differentiation of } \\
\text { naive helper T cells (Th0) into } \\
\text { Th1 cells but enhanced their } \\
\text { transition into } \\
\text { type II helper T (Th2) cells using } \\
\text { an in vitro T cell differentiation } \\
\text { assay }\end{array}$ \\
\hline Bryonia alba L. & Curcubiaceae & $\begin{array}{c}\text { derivatives of } \\
\text { trihydroxyoctade } \\
\text { cadienoic acid }\end{array}$ & roots & $\begin{array}{l}\text { Restore the disordered lipid } \\
\text { metabolism of alloxan-diabetic } \\
\text { rats }\end{array}$ \\
\hline $\begin{array}{l}\text { Bumelia } \\
\text { sartorum }\end{array}$ & Sapotaceae & bassic acid & $\begin{array}{l}\text { ethanol } \\
\text { extract of } \\
\text { root bark }\end{array}$ & $\begin{array}{l}\text { altered glucose tolerance in } \\
\text { alloxan-induced diabetic rats, } \\
\text { enhanced glucose } \\
\text { uptake in skeletal muscle and } \\
\text { significantly inhibited } \\
\text { glycogenolysis in the liver. }\end{array}$ \\
\hline $\begin{array}{l}\text { Caesalpinia } \\
\text { ferrea Mart }\end{array}$ & Leguminosae & $\begin{array}{c}\text { ellagic } \\
\text { acid and } 2^{\prime}- \\
(2,3,6- \\
\text { trihydroxy-4- } \\
\text { carboxyphenyl) } \\
\text { ellagic aci } \\
\end{array}$ & & $\begin{array}{l}\text { might contribute to the relief of } \\
\text { the long-term diabetic } \\
\text { complications }\end{array}$ \\
\hline $\begin{array}{l}\text { Camellia } \\
\text { sinensis }\end{array}$ & Theaceae & $\begin{array}{l}\text { epigallocatechin- } \\
\text { 3-O-gallate }\end{array}$ & & $\begin{array}{l}\text { may be beneficial in the } \\
\text { prevention of diabetes mellitus }\end{array}$ \\
\hline Cassia tora & Fabaceae & $\begin{array}{l}\text { a-tocopherol, } \\
\text { ascorbic acid and } \\
\text { maltodextrin }\end{array}$ & $\begin{array}{l}\text { soluble } \\
\text { fiber } \\
\text { extracted }\end{array}$ & $\begin{array}{l}\text { improve serum lipid levels in } \\
\text { type II diabetic subjects without } \\
\text { serious } \\
\text { adverse effects }\end{array}$ \\
\hline
\end{tabular}

Table 2. Important medicinal Plants used in Diabetes treatment.( continuation) 


\begin{tabular}{|c|c|c|c|c|}
\hline $\begin{array}{l}\text { Scientific } \\
\text { name }\end{array}$ & Family & $\begin{array}{c}\text { Active } \\
\text { constituent }\end{array}$ & Part Used & Folk Medical Uses \\
\hline $\begin{array}{l}\text { Cecropia } \\
\text { obtusifolia } \\
\text { Bertol }\end{array}$ & Cecropiaceae & $\begin{array}{l}\text { isoorientin and } \\
\text { 3-caffeoylquinic } \\
\text { acid }\end{array}$ & $\begin{array}{l}\text { water and } \\
\text { butanolic } \\
\text { extracts } \\
\text { prepared } \\
\text { from } \\
\text { leaves }\end{array}$ & $\begin{array}{l}\text { beneficial effects on } \\
\text { carbohydrate and lipid } \\
\text { metabolisms when it was } \\
\text { administered as an adjunct on } \\
\text { patients with type II diabetes } \\
\text { with poor response to } \\
\text { conventional medical } \\
\text { treatment. } \\
\end{array}$ \\
\hline $\begin{array}{c}\text { Cleome } \\
\text { droserifolia } \\
\text { (Capparidaceae) }\end{array}$ & & & & $\begin{array}{l}\text { possessed a postprandial } \\
\text { hypoglycemic effect but also } \\
\text { suppressed the hepatic glucose } \\
\text { release in the fasting state in a } \\
\text { comparable way to this of } \\
\text { insulin. The plant also } \\
\text { possessed hypo-cholesterolemic } \\
\text { effect, most pronounced on the } \\
\text { LDL cholesterol. }\end{array}$ \\
\hline $\begin{array}{c}\text { Cnidium } \\
\text { officinale } \\
\text { Makino }\end{array}$ & Apiaceae & & & $\begin{array}{l}\text { inhibited the high } \\
\text { glucoseinduced } \\
\text { proliferation of GMCs partially } \\
\text { through TGF-beta1 production }\end{array}$ \\
\hline Coccinia indica & Cucurbitaceae & & $\begin{array}{l}\text { ethanolic } \\
\text { extract of } \\
\text { Coccinia } \\
\text { indica } \\
\text { leaves }\end{array}$ & $\begin{array}{l}\text { normalized blood glucose and } \\
\text { caused marked improvement of } \\
\text { altered carbohydrate } \\
\text { metabolizing enzymes during } \\
\text { diabetes. }\end{array}$ \\
\hline $\begin{array}{l}\text { Commelina } \\
\text { communis }\end{array}$ & Commeliaceae & $\begin{array}{c}\text { pyrrolidine } \\
\text { alkaloid, 2,5- } \\
\text { dihydroxymethy } \\
\text { 1-3,4- } \\
\text { dihydroxypyrrol } \\
\text { idine and four } \\
\text { piperidine } \\
\text { alkaloids, 1- } \\
\text { deoxymannojiri } \\
\text { mycin, 1- } \\
\text { deoxynojirimyci } \\
\text { n, alpha- } \\
\text { homonojirimycin } \\
\text { and 7-O-beta-D- } \\
\text { glucopyranosyl } \\
\text { alpha- } \\
\text { homonojirimycin }\end{array}$ & $\begin{array}{c}\text { methanolic } \\
\text { extract }\end{array}$ & $\begin{array}{l}\text { inhibitoryactivity against } \\
\text { alpha-glucosidase }\end{array}$ \\
\hline
\end{tabular}

Table 2. Important medicinal Plants used in Diabetes treatment. (continuation) 


\begin{tabular}{|c|c|c|c|c|}
\hline $\begin{array}{l}\text { Scientific } \\
\text { name }\end{array}$ & Family & $\begin{array}{c}\text { Active } \\
\text { constituent }\end{array}$ & Part Used & Folk Medical Uses \\
\hline Croton cajucara & Euphorbiaceae & $\begin{array}{l}\text { trans- } \\
\text { hydrocortin }\end{array}$ & & $\begin{array}{l}\text { The mentioned compound also } \\
\text { effectively lowered the blood } \\
\text { sugar levels in glucose fed } \\
\text { normal rats. }\end{array}$ \\
\hline $\begin{array}{l}\text { Cryptolepis } \\
\text { sanguinolenta }\end{array}$ & Asclepiadaceae & cryptolepine & & $\begin{array}{l}\text { antihyperglycemic effect of } \\
\text { cryptolepine leaded to a } \\
\text { significant decline in plasma } \\
\text { insulin concentration, associated } \\
\text { with evidence of an } \\
\text { enhancement in insulin } \\
\text { mediated } \\
\text { glucose disposal. Finally, } \\
\text { cryptolepine increased glucose } \\
\text { uptake by 3T3-L1 cells. } \\
\end{array}$ \\
\hline $\begin{array}{l}\text { Dioscorea } \\
\text { dumetorum }\end{array}$ & Dioscoreaceae & dioscoretine & $\begin{array}{l}\text { methanol } \\
\text { extrac }\end{array}$ & $\begin{array}{l}\text { normal and alloxan diabetic } \\
\text { rabbits produced significant } \\
\text { hypoglycemic effects and the } \\
\text { hypoglycemic effects were } \\
\text { compared to those of to } \\
\text { butamide (Iwu et al., 1998) }\end{array}$ \\
\hline $\begin{array}{c}\text { Galega } \\
\text { officinalis } L .\end{array}$ & Leguminosae & Metformin & & $\begin{array}{l}\text { Treat symptoms now ascribed to } \\
\text { type II diabetes }\end{array}$ \\
\hline $\begin{array}{c}\text { Gentiana } \\
\text { olivieri Griseb }\end{array}$ & Gentianaceae & isoorientin & & $\begin{array}{l}\text { Isoorientin exhibited significant } \\
\text { hypoglycemic and } \\
\text { antihyperlipidemic effects }\end{array}$ \\
\hline $\begin{array}{l}\text { Gymnema } \\
\text { sylvestre }\end{array}$ & Asclepiadaceae & gymnemic acid I & $\begin{array}{l}\text { Water } \\
\text { soluble } \\
\text { extracts } \\
\text { the leaves }\end{array}$ & $\begin{array}{l}\text { Gymnemic acids II and III } \\
\text { showed potent inhibitory } \\
\text { activity on glucose uptake. }\end{array}$ \\
\hline $\begin{array}{l}\text { Hintonia } \\
\text { latiflora }\end{array}$ & Rubiaceae & coutaraegenin & & $\begin{array}{l}\text { coutareagenin, one of the active } \\
\text { substances contained in the } \\
\text { Hintonia latiflora bark, produces } \\
\text { a reduction of } \\
\text { the diabetic elevated blood } \\
\text { sugar levels }\end{array}$ \\
\hline $\begin{array}{l}\text { Hydnocarpus } \\
\text { wightiana }\end{array}$ & Arcariaceae & $\begin{array}{l}\text { Hydnocarpin, } \\
\text { Luteolin, } \\
\text { And } \\
\text { isohydnocarpin }\end{array}$ & $\begin{array}{l}\text { acetone } \\
\text { extract of } \\
\text { the seed } \\
\text { hulls }\end{array}$ & $\begin{array}{l}\text { the presence of antioxidant } \\
\text { molecules along with their } \\
\text { enzyme inhibitory activities in } \\
\text { the acetone extract of } \\
\text { Hydnocarpus wightiana seed } \\
\text { hulls may be responsible for the } \\
\text { antidiabetic } \\
\text { Properties }\end{array}$ \\
\hline
\end{tabular}

Table 2. Important medicinal Plants used in Diabetes treatment. (continuation) 


\begin{tabular}{|c|c|c|c|c|}
\hline $\begin{array}{l}\text { Scientific } \\
\text { name }\end{array}$ & Family & Active constituent & $\begin{array}{l}\text { Part } \\
\text { Used }\end{array}$ & Folk Medical Uses \\
\hline $\begin{array}{c}\text { Lagerstroemia } \\
\text { speciosa L. }\end{array}$ & Lythraceae & $\begin{array}{l}\text { Lagerstroemin } \\
\text { and flosin B }\end{array}$ & $\begin{array}{l}\text { hot- } \\
\text { water } \\
\text { extract } \\
\text { from } \\
\text { banaba } \\
\text { leaves }\end{array}$ & $\begin{array}{l}\text { effects on controlling of the level } \\
\text { of plasma glucose in type II } \\
\text { diabetes mellitus. }\end{array}$ \\
\hline $\begin{array}{c}\text { Larrea } \\
\text { tridentata }\end{array}$ & Zygophyllaceae & masopropol & & $\begin{array}{l}\text { lower plasma glucose } \\
\text { concentration in two mice models } \\
\text { of type II diabetes after per os } \\
\text { administration (Luo et al., 1998) }\end{array}$ \\
\hline $\begin{array}{c}\text { Myrcia } \\
\text { multiflora }\end{array}$ & Myrtaceae & myrciacitrin I & $\begin{array}{l}\text { ethyl } \\
\text { acetate- } \\
\text { soluble } \\
\text { portion, }\end{array}$ & $\begin{array}{l}\text { show inhibitory activities on } \\
\text { ALR2 and alpha-glucosidase as } \\
\text { well as on the increase of serum } \\
\text { glucose level in sucrose-loaded } \\
\text { rats and in alloxan-induced } \\
\text { diabetic mice (Yoshikawa et al., } \\
\text { 1998). }\end{array}$ \\
\hline $\begin{array}{c}\text { Paeonia } \\
\text { lactiflora Pall. }\end{array}$ & Panunculaceae & paeoniflorin & & $\begin{array}{l}\text { the mentioned glucoside reduced } \\
\text { the elevation of blood sugar in } \\
\text { glucose challenged rats. Increase } \\
\text { of glucose utilization by } \\
\text { paeoniflorin can thus be } \\
\text { considered (Hsu et al., 1997). }\end{array}$ \\
\hline $\begin{array}{l}\text { Pandanus } \\
\text { odorus Ridl. }\end{array}$ & Pantadaceae & $\begin{array}{l}\text { 4-hydroxybenzoic } \\
\text { acid }\end{array}$ & & $\begin{array}{l}\text { 4-hydroxybenzoic acid caused a } \\
\text { decrease in plasma glucose levels } \\
\text { dose-dependently. The compound } \\
\text { did not affect serum insulin level } \\
\text { and liver glycogen content in the } \\
\text { diabetic model, but increased } \\
\text { glucose consumption in normal } \\
\text { and diabetic rat diaphragms. }\end{array}$ \\
\hline $\begin{array}{l}\text { Phyllanthus } \\
\text { sellowianus }\end{array}$ & Euphorbiaceae & $\begin{array}{l}\text { isoqu ercitrin and } \\
\text { rutin }\end{array}$ & & $\begin{array}{l}\text { hypoglycemic and diuretic agent } \\
\text { reduction in blood } \\
\text { glucose levels }\end{array}$ \\
\hline $\begin{array}{c}\text { Pueraria } \\
\text { thunbergiana }\end{array}$ & Leguminosae & kaikasaponin III & & $\begin{array}{l}\text { kaikasaponin III (as a mixture of } \\
\text { isomers), may exhibit its } \\
\text { hypoglycemic and hypolipidemic } \\
\text { effects by up-regulating or down- } \\
\text { regulating } \\
\text { antioxidant mechanisms via the } \\
\text { changes in Phase I and II enzyme } \\
\text { activities. }\end{array}$ \\
\hline $\begin{array}{l}\text { Stevia } \\
\text { rebaudiana } \\
\text { Bertoni }\end{array}$ & Compositae & stevioside & & $\begin{array}{l}\text { Stevioside was able to regulate } \\
\text { blood glucose levels by enhancing } \\
\text { not only insulin secretion, but also } \\
\text { insulin utilization in insulin- } \\
\text { deficient rats; the latter was due to } \\
\text { decreased PEPCK gene expression } \\
\text { in rat liver by stevioside's action } \\
\text { of slowing down Gluconeogenesis } \\
\text { (Chen et al., 2005). }\end{array}$ \\
\hline
\end{tabular}

Table 2. Important medicinal Plants used in Diabetes treatment. (continuation) 
<smiles>O=c1c(O)c(-c2cc(O)c(O)c(O)c2)oc2cc(O)cc(O)c12</smiles><smiles>C=CCSS(=O)CC=C</smiles>

allic ine<smiles>O=c1c2cc(O)c(O)cc2oc2cc(O)c([C@@H]3O[C@H](CO)C(O)C(O)[C@H]3O)c(O)c12</smiles>
mangiferin<smiles>C=C(c1c(O)c(CC=C(C)C)c(O)c2c1oc1c(CC(O)C(=C)C)c(O)c(C(=O)C(C)CC)c(O)c12)C(C)CC</smiles>

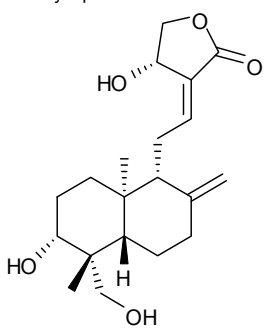

andrographolide

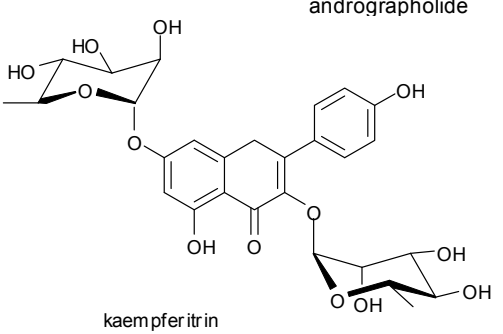

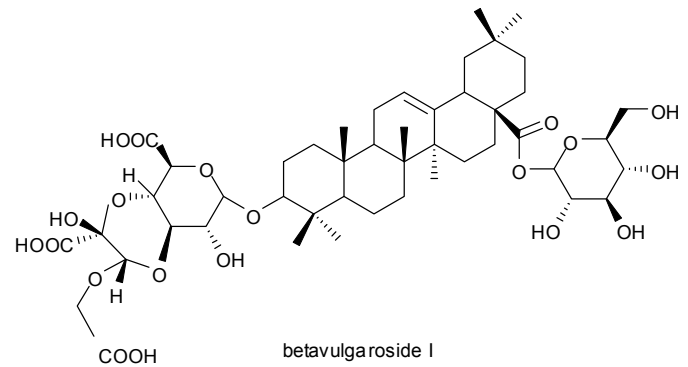

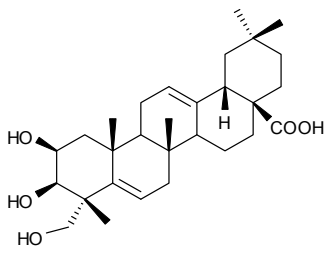

bassic acid

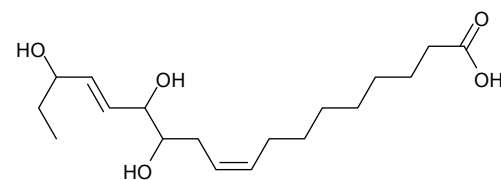

12,13,16-trihyd roxy-9Z,14E-octadecadienoic acid<smiles>O=c1oc2c(O)c(O)cc3c(=O)oc4c(O)c(O)cc1c4c23</smiles>

ellagic acid

Fig. 1. Some important active compound involved in diabetes mellitus treatment. 
<smiles>O=C(OC1Cc2c(O)cc(O)cc2OC1c1ccc(O)c(O)c1)c1cc(O)c(O)c(O)c1</smiles>

epiga llocatechin-3-O-g alla te<smiles>CN1C2=C3C=CC=CC3N=C2C=C2C=CC=CC21</smiles>

cryptolepine<smiles>CC(Cc1ccc(O)c(O)c1)C(C)Cc1ccc(O)c(O)c1</smiles>

masopropol<smiles>CN(C)C(=N)NC(=N)N</smiles><smiles>O=c1cc(-c2cc(O)c(O)c(O)c2)oc2cc(O)c(C3OCC4OC3C(O)C(O)C(O)C4O)c(O)c12</smiles>

isoo rientin<smiles>CC(CC(=O)O)CC1(O)CC2CCC1N2C</smiles>

dioscoretine<smiles>C/C=C\CCCCCCCCCC1=CC(=O)C=C(OC)C1=O</smiles>

maesanin

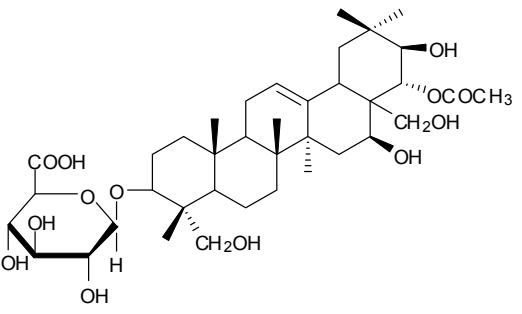

gymnemic acid I

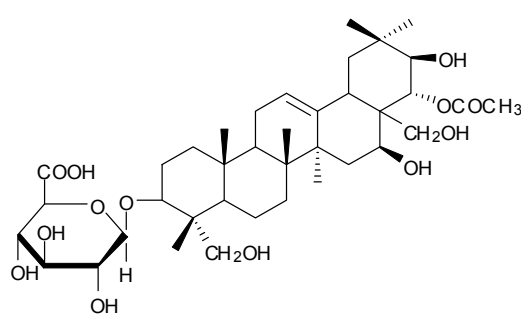

gymnemic acid II

Fig. 1. Some important active compound involved in diabetes mellitus treatment. (continuation) 
<smiles>COc1cc(C2Oc3ccc(-c4cc(=O)c5c(O)cc(O)cc5o4)cc3OC2CO)ccc1O</smiles>

hydnocarpin<smiles>O=c1cc(-c2ccc(O)c(O)c2)oc2cc(O)cc(O)c12</smiles>

luteolin<smiles>COc1cc(C2Oc3c(O)cc(-c4cc(=O)c5c(O)cc(O)cc5o4)cc3C2CO)ccc1O</smiles>

isoh ydnocarpin<smiles>Cc1c(O)c(C)c2c(c1O)C(=O)C[C@@H](c1cc(O)ccc1O)O2</smiles>

myrciacitrin I<smiles>CC(=O)c1c(O)cc(O)cc1OC1OC2OC(C1O)C(O)C(O)C2O</smiles>

myrciaphenon A

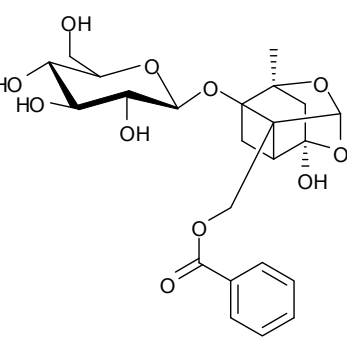

paeoniflorin<smiles>COc1ccc(O)c([C@@H]2CC(=O)c3c(O)c(C)c(OC4OC(CO)C5O[C@H](O)C(O)[C@H]4O5)c(C)c3O2)c1</smiles>

myrciacitrin II<smiles>CC(=O)c1c(O)cc(O)cc1OC1OC2COC(O)C(O)C(O1)C(O)C2OC(=O)c1cc(O)c(O)c(O)c1</smiles>

myrciaphenon B

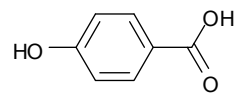

4-hydroxybenzoic acid

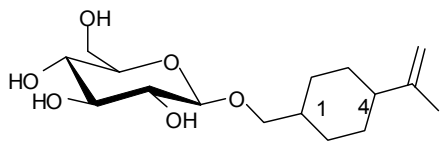

perilloside C:1,4-trans perilloside D:1,4-cis

Fig. 1. Some important active compound involved in diabetes mellitus treatment. (continuation) 


\section{Some biomedical application for some plant used in treating hypoglycemic and hyperglycemic conditions}

\section{A- Amelioration of hyperglycemia in STZ-diabetic rats.}

Several approaches were conducted in order to investigate the hypoglycemic and hypolipidemic activities of plant extract on experimental animals (STZ-diabetic rats). The derived data showed that some extracts are potent in the amelioration of hyperglycemia in STZ-diabetic rats and is a potential source for the isolation of new per os active agent(s) for anti-diabetic therapy like what observed in Averrhoa bilimbi ethanolic extract which has hypoglycemic, hypotriglyceridemic, anti-lipid peroxidative and anti-atherogenic properties in STZ-diabetic rats (Purshparaj et al., 2001). Another way was by the inhibition of myeloperoxidase activity and decreased lipid peroxidation, induced by ascorbyl radical either in microsomes or in asolectin and phosphatidylcholine liposomes as what observed in Bauhinia forficate due to the kaempferitrin effect that showed high reactivity with the free radical 1,1-diphenyl-2-picryl hydrazyl (DPPH),

In the plant Beta vulgaris L. var. cicla (Chenopodiaceae) the anti-diabetic effect in experimental animals may be to the reduction in blood glucose levels by the regeneration of the $\beta$ cells (Bolkent et al., 2000). Betavulgarosides I, II, III, IV and oleanolic acid oligoglycosides produced hypoglycemic effects that was demonstrated by a per os glucose tolerance test in rats after their per os administration (Yoshikawa et al., 1996). Or through stimulating the synthesis/release of insulin from the $\beta$ cells of Langerhans like the effect of Biophytum sensitivum and/or also mediated through enhance secretion of insulin from the $\beta$ cells of Langerhans or through an Extra pancreatic mechanism (Catharanthus roseus L. (Apocynaceae)). Or by increasing the number of islet $\beta$ cells and that of islet a cells was decreased in STZ-diabetic rats like what happen in Dendrobium candidum (Orchidaceae), Its mechanism of action probably involves the stimulation of the secretion of insulin from $\beta$ cells and the inhibition of the secretion of glucagons from $\alpha$ cells and it can probably decrease the decomposition of liver glycogen and increase the synthesis of liver glycogen.

The Administration of Coccinia indica (Cucurbitaceae) leaf extract, an indigenous plant used in Ayurvedic medicine in India, to normal and STZ-diabetic animals exhibited significant hypoglycemic and antihyperglycemic effect and reversed the associated with diabetes biochemical alterations (Venkateswaran \& Pari 2002). The results indicated that the per os administration of Coccinia indica leaf extract to diabetic animals normalized blood glucose and caused marked improvement of altered carbohydrate metabolizing enzymes during diabetes. The antioxidant effects of an ethanolic extract of Coccinia indica leaves was studied in STZ-diabetic rats (Venkateswaran \& Pari 2003). Per os administration of Coccinia indica leaf extract resulted in a significant reduction in thiobarbituric acid reactive substances and hydroperoxides and a significant increase in reduced GSH, SOD, CAT, glutathione peroxidase and glutathione-S-transferase in liver and kidney of STZ-diabetic rats, which clearly showed the extract's antioxidant property. The ethnopharmacological use of Gongronema latifolium in ameliorating the oxidative stress found in diabetics and indicating promise of possible use in lessening morbidity in affected individuals.

The obtained result by Kim et al 2006 suggest that the administration of Chrysanthemum coronarium and Morus alba, have a hypoglycemic effect in diabetic rats and their effect was 
equivalent to that of glibenclamide. The administration of C.unshiu shows more antihyperlipidemic effect than antidiabetic effect. The effect of aqueous extracts from four medicinal plants on the blood glucose levels of experimental animals was determined at various time intervals for $9 \mathrm{~h}$ after oral administration at $100 \mathrm{mg}$ dose kg- $1 \mathrm{~b} . w \mathrm{t}$. (Fig. 2). There was a significant elevation in the blood glucose level by 3.3-5 times during experimental time period in alloxan-induced diabetic rats, when compared to normal rats. The administration of $C$. coronarium extract caused the blood glucose levels of diabetic rats to $83.4,67.6,75.1,81.1$ and $74.3 \%$ at the time interval of $1,3,5,7$ and $9 \mathrm{~h}$, respectively $(\mathrm{p}<0.05)$. Maximum reduction of $32.4 \%$ was observed $3 \mathrm{~h}$ after treatment. The administration of $M$. alba extract produced the most significant reduction $(\mathrm{p}<0.05)$ among four medicinal plants in the blood glucose levels of $34,41,33$ and $35 \%$ at 3, 5, 7 and $9 \mathrm{~h}$ respectively.

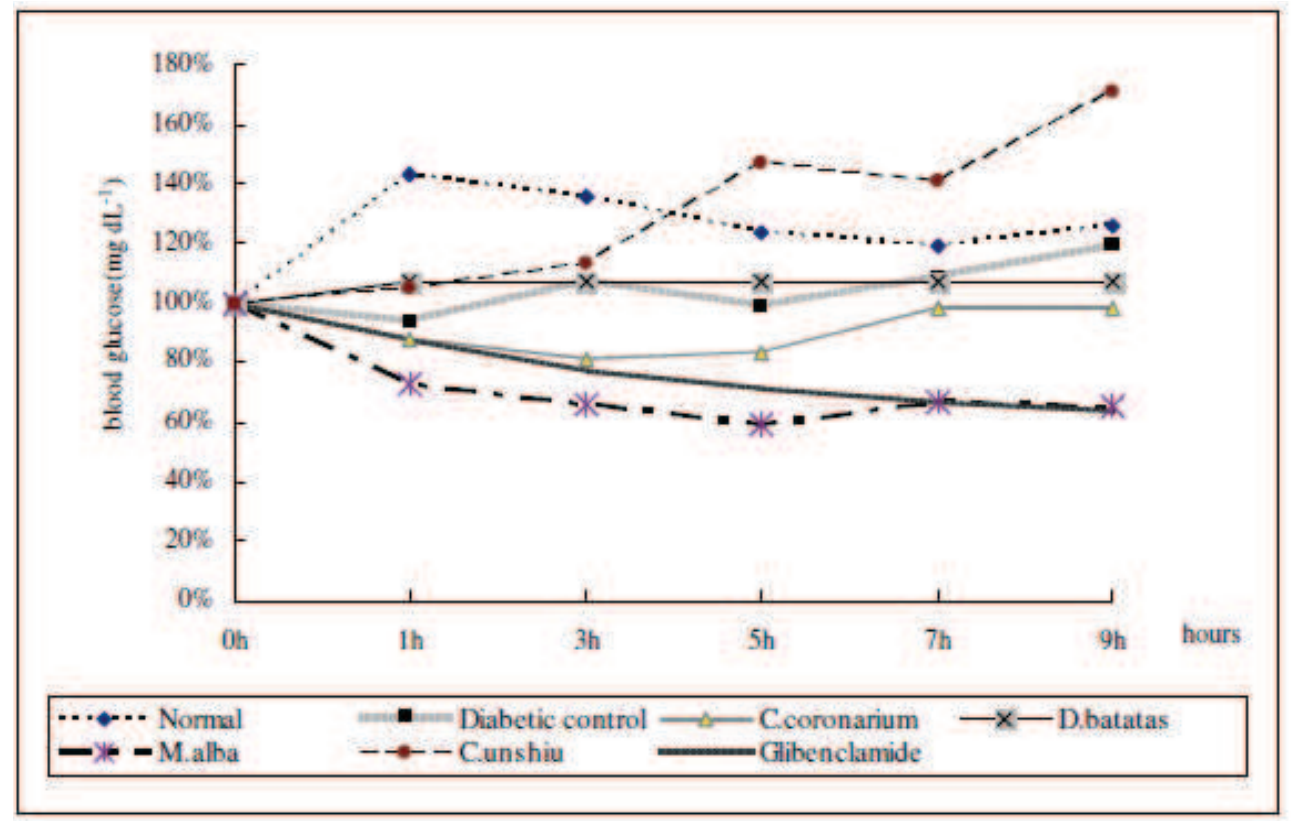

Fig. 2. Percentage of effect of experimental plants on plasma glucose levels compared with 0 $\mathrm{h}$ data in alloxaninduced diabetic rats (\%). Values are mean percent of blood glucose concentration $(n=6)$. According to the study obtained by Kim et al., 2006.

\section{B- Hypoglycemic, hypolipidemic hypotriglyceridemic, anti-lipid peroxidative and anti- atherogenic properties of these agents in different medicinal plant:}

Insulin resistance (hyperinsulinemia) is now recognized as a major contributor to the development of glucose intolerance, dyslipidemia and hypertension in type II diabetic patients. Aqueous extracts of Pterocarpus marsupium bark (Fbaceae) and Trigonella foenum-graecum. (Leguminosae) seeds have been shown to exert hypoglycemic/antihyperglycemic effect in experimental as well as in clinical settings (Grover et al., 2005). Results of this study, in addition to previous clinical benefits of 
Pterocarpus marsupium seen in type II diabetic subjects, are suggestive of usefulness of its bark in insulin resistance, the associated disorder of type II diabetes. Though several antidiabetic principles (epicatechin, pterosupin, marsupin and pterostilbene) have been identified.

The administration of trihydroxyoctadecadienoic acid and its derivatives obtained from the roots of the native Armenian plant Bryonia alba (Curcubiaceae) was found to restore the disordered lipid metabolism of alloxan-diabetic rats (Karageuzyan et al., 1998). These derivatives of trihydroxyoctadecadienoic acid can correct major metabolic abnormalities typical of severe diabetes mellitus they can influence the profile of the formation of stable prostaglandins by actions such as downstream of prostaglandin endoperoxides. In Camellia sinensis (Theaceae) species, the blood glucose lowering activity of Camellia sinensis was studied by many workers. It has recently been reported that the major green tea polyphenolic constituent, epigallocatechin 3- gallate, mimics the cellular effects of insulin including the reductive effect on the gene expression of rate-limiting gluconeogenic enzymes in a cell culture system (Koyama et al., 2004). Per os administration of green tea that contains this polyphenolic constituent caused a reduction in the levels of mRNAs for gluconeogenic enzymes, PEPCK and G6Pase in the mouse liver. Epigallocatechin 3-gallate alone was also found to down-regulate the gene expression of these enzymes in vitro.

Bumelia sartorum (Sapotaceae), the hypoglycemic effect may be similar to chlorpropamide and possibly due to an enhanced secretion of insulin from the islets of Langerhans or an increased utilization of glucose by peripheral tissues. Besides hypoglycemic activity, the ethanol extract also elicited significant anti-inflammatory activity, but did not show any significant effects on blood pressure, respiration or on the various isolated tissue preparations studied. Bassic acid, an unsaturated triterpene acid isolated from an ethanol extract of Bumelia sartorum root bark, elicited significant hypoglycemic activity in alloxandiabetic rats and altered the pattern of glucose tolerance in these animals when administered per os (Naik et al., 1991). In addition, bassic acid treatment increased significantly the glucose uptake process and glycogen synthesis in isolated rat diaphragm. Bassic acid treatment increased plasma insulin levels significantly in alloxandiabetic rats. It was therefore suggested that the hypoglycemia activity of bassic acid may be mediated through enhanced secretion of insulin from the pancreatic $\beta$ cells.

Compelling evidence of Bidens pilosa (Asteraceae) suggests that infiltrating CD4 type I helper $\mathrm{T}$ (Th1) cells in the pancreatic islets play a pivotal role in the progression of diabetes in nonobese diabetic mice. Treatment with a butanol fraction of Bidens blood glucose and insulin in non-obese diabetic mice in a dose-dependent manner and elevated the serum IgE levels regulated by Th2 cytokines (Chang et al., 2004). Moreover, the butanol fraction inhibited the differentiation of naive helper T cells (Th0) into Th1 cells but enhanced their transition into type II helper T (Th2) cells using an in vitro T cell differentiation assay. The butanol fraction of Biderns pilosa and its polyacetylenes can prevent diabetes possibly via suppressing the differentiation of Th0 cells into Th1 cells and promoting that of Th0 cells into Th2 cells.

The results of a reported study of Gongronema latifolium (Asclepiadaceae) suggest that the extracts from leaves could exert their antidiabetic activities through their antioxidant properties (Ugochukwu \& Bababy 2002). The aqueous and ethanolic extracts were tested in order to evaluate their effect on renal oxidative stress and lipid peroxidation in non-diabetic 
and STZ-diabetic rats after per os administration (Ugochukwu \& Makini 2003). The ethanolic extract appeared to be more effective in reducing oxidative stress, lipid peroxidation, and increasing the GSH/GSSG ratio, The antihyperglycemic effects of aqueous and ethanolic extracts from Gongronema latifolium leaves was also investigated on glucose and glycogen metabolism in liver of non-diabetic and STZ-diabetic rats (Ugochukwu \& Bababy 2003). The data showed that the ethanolic extract from the plant's leaves had antihyperglycemic potency, which was suggested to be mediated through the activation of HK, PFK, G6PD and inhibition of GK in the liver.

According to the study occurred by Mohammad Khalil, et al. (2010), it is found found that, the treatment of the diabetic rats with Citrullus colocynthis pulp extract, in the livers showed, more or less, an improvement in the histological architecture with persistence of the cytoplasmic vacuoles in some hepatocytes that could be attributed to the residual adverse effect of the diabetic affliction. But the noticed apparent general improvement signifies that Citrullus colocynthis could possess cyto-protective abilities on the hepatocytes. The present findings are supported by those announced by Aburjai et al. (2007) who confirmed the antidiabetic properties of Citrullus colocynthis extract. Also, Sebbagh et al. (2009) stated that this plant could improve the streptozotosin-induced diabetes in rats. Bujanda et al. (2008) owed the similar steatotic inhibitory effect of resveratol to inhibition of the tumor necrosis factor alpha production, lipid per-oxidation and oxidative stress.

The damaged sinusoids, the aemorrhage and the inflammatory cell infiltration encountered in the liver of the diabetic animals, might be due to the hyperglycemic state. Seifalian et al. (1999), in an analogous study in rabbits, stressed on that the sinusoidal affection is correlated with the severity of fat accumulation in the parenchymal cells. According to the findings of Khan and Chakarabarti (2003); Hayden et al. (2005) and Ban and Twigg (2008), hyperglycemia is the main offending factor in the onset of the micro-vascular diabetic complications. Fortunately, following Citrullus colocynthis intake, in their study, the damaged sinusoids, the haemorrhage and the inflammatory cell infiltration subsided indicating a beneficial role of such a remedy. These findings are supported by those claimed by Alarcon-Aguilar et al. (2000). Despite the obvious antidiabetic effect of Citrullus colocynthis pulp extract, its use in normal rats, in the current work, caused hepatocytic poration and few hapatocytes had condensed or fragmented nuclei indicating minimal hepatotoxicity.

This herbal therapy of Gymnema sylvestre (Asclepiadaceae) appears to bring about blood glucose homeostasis through increased serum insulin levels provided by repair/regeneration of the endocrine pancreas. Also, the effectiveness of the water extract from the leaves of Gymnema sylvestre, in controlling hyperglycemia was investigated in type II diabetic patients as conventional per os anti-hyperglycemic agents (Baskaran et al., 1990). The obtained data suggested that the $\beta$ cells may be regenerated / repaired in type II diabetic patients on the extract's supplementation. This was supported by the raised insulin levels in the serum of patients after the supplementation. Furthermore, the antihyperglycemic action of a crude saponin fraction and five triterpene glycosides (gymnemic acids I-IV and gymnemasaponin V) derived from the methanol extract of leaves of Gymnema sylvestre was investigated in STZ-diabetic mice (Sugihara et al., 2000).

The results indicated that insulin-releasing action of gymnemic acid IV, administered i.p., may contribute to the antihyperglycemic effect by the leaves of Gymnema sylvestre. 

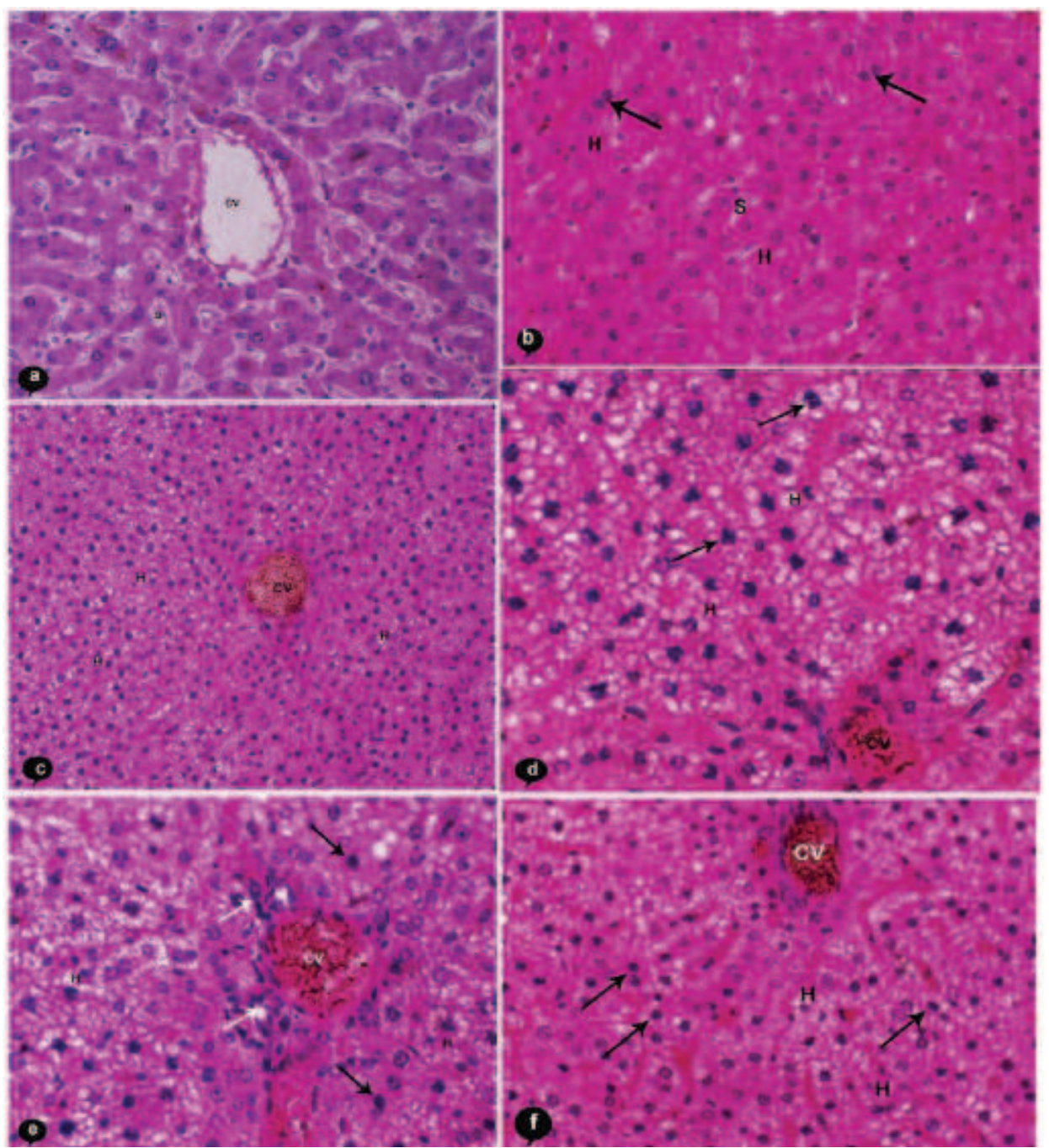

Fig. 3. Photomicrographs of the livers of control, Citrullus colocynthis- treated, diabetic and Citrullus colocynthistreated diabetic rats, H\&E stain; (a) Liver of control rats, $\times 40$; (b) Liver of Citrullus colocynthis treated rats, showing few hapatocytes had condensed or fragmented nuclei $\times 40$; (c) Liver of diabetic rats, showing disorganized hepatic cords, reduced sinusoids and many hepatocytes having cytoplasmic vacuolization, $\times 20$; (d) Higher magnification of (c), showing most hepatocytes with cytoplasmic vacuolar degeneration and pyknotic nuclei (arrows), $\times 40$; (e) Liver diabetic rats, showing a central inflammatory cell infiltration (white arrows) and hepatocytes with cytoplasmic vacuolar degeneration and pyknotic nuclei (black arrows), $\times 40$; (f) Liver of diabetic rats treated with Citrullus colocynthis, showing recovered hepatocytes with less cytoplasmic vacuolization compared with diabetic animals. Few hepatocytes with cytoplasmic vacuolization and pyknotic nuclei are still seen (arrows), $\times 40$. According to the study occurred by Mohammad Khalil, et al. (2010). 


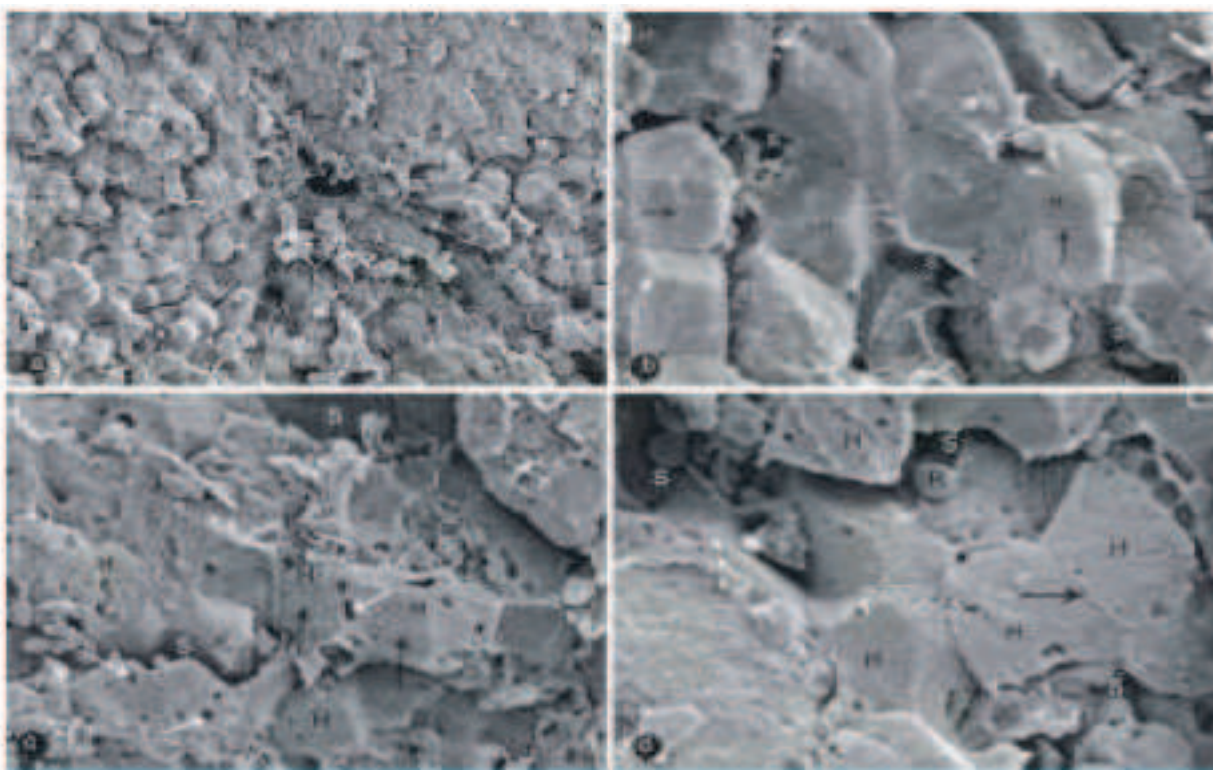

Fig. 4. Scanning electron micrographs of livers of control and Citrullus colocynthis-treated rats. (a) SEM of the liverof control rats showing normal hepatic cord around a Central Vein (CV), $\times 450$; (b) Larger magnification of (a) showing normal Sinusoids (S) and normal Hepatocytes $(\mathrm{H})$ with normal intercellular boundaries (arrows), $\times 1500$ (c); SEM of the liver of Citrullus colocynthis-treated rats, showing rows of Hepatocytes $(\mathrm{H})$ containing few pores (white arrows). Note the blood Sinusoids (S) and the intercellular boundaries (black arrows), $\times 1100$; (d) Higher magnification of (c), showing blood Sinusoids (S), Hepatocytes (H), intercellular spaces (black arrows) and erythrocytes (R), $\times 1500$. According to the study occurred by Mohammad Khalil, et al. (2010).

Gymnemic acid IV may be an anti-obese and antihyperglycemic prodrug. The inhibitory activity of each triterpene glycoside on the glucose uptake in rat small intestine fragments was examined, in order to determine its impact on the increase of serum glucose level in glucose-loaded rats (Yoshikawa et al., 1997). It was found that Gymnemic acids II and III showed potent inhibitory activity on glucose uptake. Gymnemoside b and gymnemic acids III, V, VII were found to exhibit a little inhibitory activity against glucose uptake, and the principal constituents, gymnemic acid I and gymnemasaponin V, lacked this activity. It is noteworthy that, although Gymnema saponin constituents such as Gymnemic acids II and III show no effect on serum glucose levels in oral-loaded rats, they exhibit potent inhibitory activity on the glucose uptake and further studies need to be contacted.

The hypoglycemic activity of a decoction from Juniperus communis (juniper berries) both in normal glycemic and in STZ-diabetic rats was studied (Sanchez et al., 1994). Juniper decoction decreased glycemic levels in normal glycemic rats through an increase of peripheral glucose utilization or a potentiation of glucose induced insulin secretion.

The per os administration of the decoction to STZ diabetic rats resulted in a significant reduction both in blood glucose levels and in the mortality index, as well as the prevention 

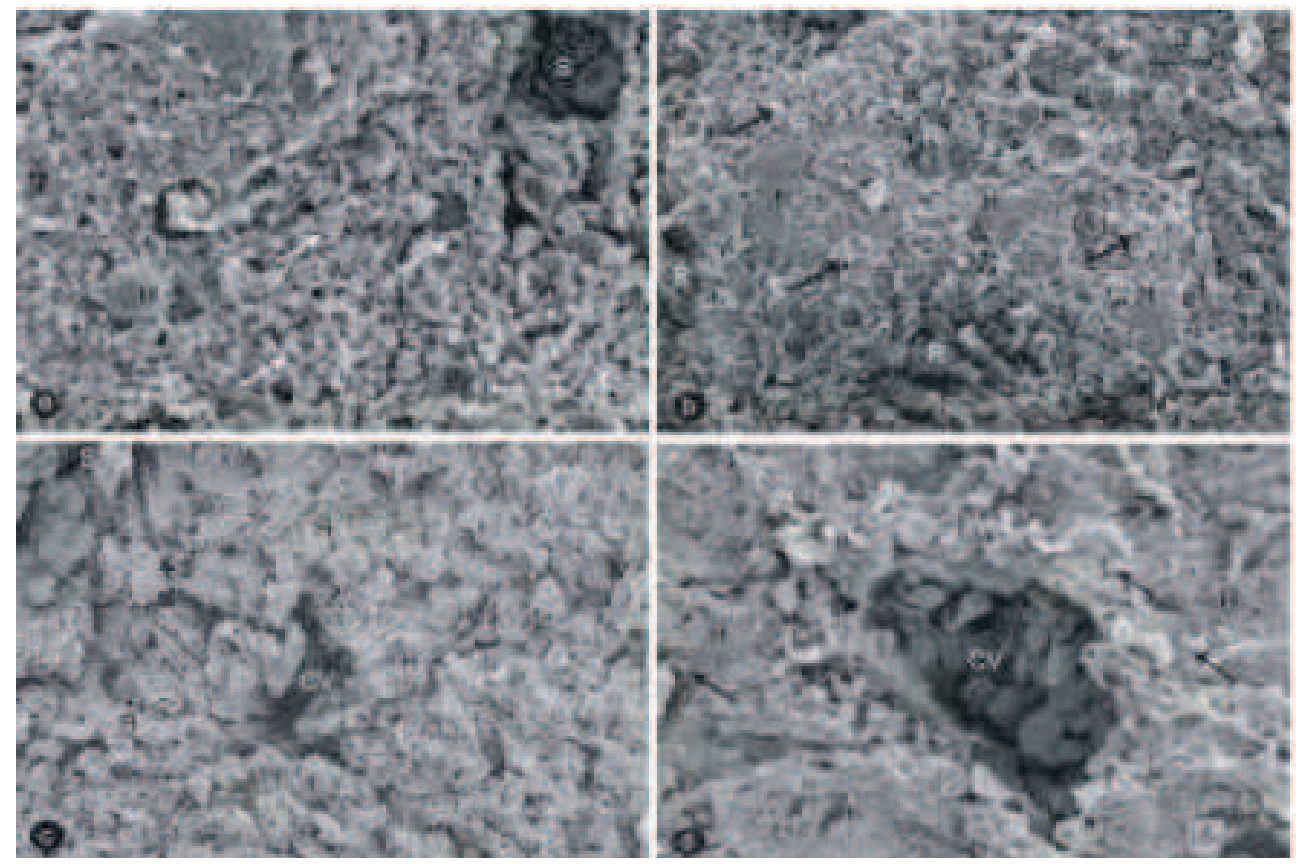

Fig. 5. Scanning electron micrographs of livers of diabetic and Citrullus colocynthis-treated diabetic rats. (a) SEM of the liver of diabetic rats showing degenerating Hepatocytes $(\mathrm{H})$ having a plenty of lipid droplets (white arrows). Damaged blood Sinusoids (S) are also seen as well as some erythrocytes $(R)$ are spread inside hepatocytes, $\times 450$; (b) SEM of the liver of diabetic rats showing hemorrhage of erythrocytes $(\mathrm{R})$ between Hepatocytes $(\mathrm{H})$. Lipid degeneration (black arrows) of hepatocytes is also seen, $\times 450$; (c) SEM of the liver of Citrullus colocynthis-treated diabetic rats, showing intact Hepatocytes $(\mathrm{H})$ and blood Sinusoids (S). Only fewhepatocytes around the central vein have some vacuoles, $\times 450$; (d) SEM of the liver of Citrullus colocynthis treated diabetic rats $(300 \mathrm{mg})$ with higher magnification, showing intact Hepatocytes $(\mathrm{H})$ and a Central Vein $(\mathrm{CV})$ containing a lot of erythrocytes. A few hepatocytes around the central vein have a few lipid droplets (arrows), $\times 1100$. According to the study occurred by Mohammad Khalil, et al. (2010).

of the loss of body weight. This effect seemed to be mediated by the peripheral action of juniper while in Olea europaea (Oleaceae) plant species, the hypoglycemic activity may result from two mechanisms: (a) potentiation of glucose induced insulin release, and (b) increased peripheral uptake of glucose.

The hypoglycemic effect of the rhizomes of Polygala senega (Polygalaceae) was proposed that after i.p. administration was without altering the insulin levels and with the need of the presence of insulin in order to act. In addition, one of the active components of this hypoglycemic effect was identified as a triterpenoid glycoside, senegin-II (as a mixture of isomers). Solanum lycocarpum (Solanaceae) has been widely employed for diabetes management, obesity and to decrease cholesterol levels. Some of the polysaccharides slowed gastric emptying and act on the endocrinous system affecting the liberation of 
gastrointestinal hormones, lowering blood glucose levels. The hypocholesterolemic activity could be due to the increased fecal bile acid excretion as well as to the action of the shortchain fatty acids, coming from fermentation, on the synthesis of deltaaminolevulinate and by the increase of the cholesterol 7-alpha-hydroxylase and 3-hydroxy-3-methylglutaryl CoA reductase synthesis (DalAgnol \& Lino von Poser 2000).

\section{Conclusions and future directions}

Scientists from divergent fields are investigating plants anew with an eye to their antimicrobial usefulness. A sense of urgency accompanies the search as the pace of species extinction continues. Laboratories of the world have found literally thousands of phytochemicals which have inhibitory effects on all types of microorganisms in vitro. More of these compounds should be subjected to animal and human studies to determine their effectiveness in whole-organism systems, including in particular toxicity studies as well as an examination of their effects on beneficial normal microbiota. It would be advantageous to standardize methods of extraction and in vitro testing so that the search could be more systematic and interpretation of results would be facilitated. Also, alternative mechanisms of infection prevention and treatment should be included in initial activity screenings. Disruption of adhesion is one example of an anti-infection activity not commonly screened for currently. Attention to these issues could usher in a badly needed new era of chemotherapeutic treatment of infection by using plant-derived principles.

\section{References}

Aburjai, T., M. Hudaib, R. Tayyem, M. Yousef and M. Qishawi, 2007. Ethnopharmacological survey of medicinal herbs in Jordan, the Ajloun heights egion. J. Ethnopharmacol., 110: 294-304. PMID: 7097250

Alarcon-Aguilar, F.J., M. Jimenez-Estrad, R. Reyes- Chilpa and R. Roman-Ramos, 2000. Hypoglycemic effect of extracts and fractions from Psacalium decompositum in healthy and alloxan-diabetic mice. J. Ethnopharmacol., 72:

Arise, R.O., S.O. Malomo, J.O. Adebayo and A. Igunnu, 2009. Effects of aqueous extract of eucalyptus globules on lipid peroxidation and selected enzymes of rat liver. J. Med. Plants Res., 3: 77-81

Brixova, E., 1981. Experimental and clinical liver steatosis. Folia Fac. Med. Univ. Comenian Bratisl., 19: 9-90.

Ban, C.R. and S.M. Twigg, 2008. Fibrosis in diabetes complications: Pathogenic mechanisms and circulating and urinary markers. Vasc. Health Risk Manage., 4: 575-596. PMID: 18827908

Bolkent S, Yanardag R, Tabakoglu-Oguz A, Ozsoy-Sacan A. “Effects of chard (Beta bulgaris L. var. cicla) extract on pancreatic B cells in diabetic rats: a morphological and biochemical study". J. Ethnopharmacol. 2000; 73: 251-259.

Baskaran K, Kizar Ahamath B, Radha Shanmugasundaram K, ShanmugasundaramER. "Antidiabetic effects of a leaf extract from Gymnema sylvestre in non- 
insulin-dependent diabetes mellitus patients". J. Ethnopharmacol. 1990; 30(3): 295300 .

Bujanda, L., E. Hijono, M. Larzabal, M. Beraza and P. Aldazabal et al., 2008. Resveratrol inhibits non-alcoholic fatty liver disease in rats. BMC Gastroenterol., 8: 40-40. DOI: 10.1186/1471-230X-8-40

Chang SL, Chang CL, Chiang YM, Hsieh RH, Tzeng CR, Wu TK, Sytwu HK, Shyur LF, Yang WC. "Polyacetylenic compounds and butanol fraction from Bidens pilosa can modulate the differentiation of helper $\mathrm{T}$ cells and prevent autoimmune diabetes in non-obese diabetic mice". Planta Med. 2004; 70(11): 10451051.

Chen TH, Chen SC, Chan P, Chu YL, Yang HY, Cheng JT. “ Mechanism of the hypoglycemic effect of stevioside, a glycoside of Stevia rebaudiana". Planta Med. 2005; 71(2): 108113. Dall'Agnol R, Lino von Poser G. "The use of complex polysaccharides in the management of metabolic diseases: the case of Solanum lycocarpum fruits". J. Ethnopharmacol. 2000; 71(1-2): 337-341.

Fagot-Campagna A. \& Narayan K. “Type 2 diabetes in children”. Br. Med. J. 2001: 322: 377387.

Fugh-Beerman A. "Herb-drug interactions". Lancet 2000; 355: 134-138.

Fujisawa T, Ikegami H, Kawaguchi Y, Nojima K, Kawabata Y, Ono M, Nishino M, Noso S. Taniguchi H, Horiki M, Itoi-Babaya M, Babaya N, Inoue K, Ogihata T. "Common genetic basis between type I and type II diabetes mellitus indicated by interview-based assessment of family history ". Diabetes Res. Clin. Pract. 2004; 66S:S91-S95

Ghosh, S. and S.A. Surawanshi, 2001. Effect of Vinca rosea extracts in treatment of alloxan diabetes in male albino rats. Indian J. Exp. Biol., 39: 748759.PMID:12018575

Grover JK, Vats V, Yadav SS. “Pterocarpus marsupium extract (Vijayasar) prevented the alteration in metabolic patterns induced in the normal rat by feeding an adequate diet containing fructose as sole carbohydrate". Diabetes Obes. Metab. 2005; 7(4): 414417.

Huxtable RJ. "The harmfull potential of herbal and other plant products". Drug Safety 1990; 5(Suppl. 1): 126-136.

Hsu FL, Lai CW, Cheng JT. "Antihyperglycemic effects of paeoniflorin and 8debenzoylpaeoniflorin, glycosides from the root of Paeonia lactiflora". Planta Med. 1997; 63(4): 323-325.

Hayden, M.R., J.R. Sowers and S.C. Tyagi, 2005. The central role of vascular extracellular matrix and basement membrane remodeling in metabolic syndrome in type and 2 diabetes: The matrix preloaded. Cardiovasc. Diabetol., 4: 9-9. DOI: 10.1186/14752840-4-9

Khan, Z.A. and S. Chakarabarti, 2003. Endothelins in chronic diabetic complications. Can. J. Physiol. Pharmol., 81: 622-634. DOI: 10.1139/Y03-053

Keith, K.G., V. Fonseca, M.H. Tan and A. Dalpiaz, 2004. Narrative review: Hepatobiliary disease in type 2 diabetes mellitus. Ann. Intern. Med., 141: 946-956. PMID: 15611492 
Koyama Y, Abe K, Sano Y, Ishizaki Y, Njelekela M, Shoji Y, Hara Y, Isemura M. "Effects of green tea on gene expression of hepatic gluconeogenic enzymes in vivo". Planta Med. 2004; 70(11): 1100-1102.

Karageuzyan KG, Vartanyan GS, Agadjanov MI, Panossian AG, Hoult JR. "Restoration of the disordered glucose-fatty acid cycle in alloxan-diabetic rats by trihydroxyoctadecadienoic acids from Bryonia alba, a native Armenian medicinal plant". Planta Med. 1998; 64(5): 417-422.

Ji Su Kim, Jung Bong Ju , Chang Won Choi and Sei Chang Kim (2006): Hypoglycemic and Antihyperlipidemic Effect of Four Korean Medicinal Plants in Alloxan Induced Diabetic Rats American Journal of Biochemistry and Biotechnology 2 (4): 154-160, 2006 ISSN 1553-3468

Luo J, Chuang T, Cheung J, Quan J, Tsai J, Sullivan C, Hector RF, Reed MJ, Meszaros K, King SR, Carlson TJ, Reaven GM. "Masoprocol (nordihydroguaiaretic acid): a new antihyperglycemic agent isolated from the creosote bush (Larrea tridentata)". Eur. J. Pharmacol. 1998; 346(1): 77-79.

Luo J, Fort DM, Carlson TJ, Noamesi BK, nii-Amon-Kotei D, King SR, Tsai J, Quan J, Hobensack C, Lapresca P, Waldeck N, Mendez CD, Jolad SD, Bierer DE, Reaven GM. " Cryptolepis sanguinolenta: an ethnobotanical approach to drug discovery and the isolation of a potentially useful new antihyperglycemic agent". Diabet. Med. 1998; 15(5): 367-374.

Lyra, R., M. Oliveira, D. Lins and N. Cavalcanti, 2006. Prevention of type 2 diabetes mellitus. Arq. Bras. Endocrinol. Metabo,. 50: 239-249.

MacFarlance IA, Bliss M, Jackson JG, Williams G. "The history of diabetes mellitus. In textbook of diabetes". (eds Pichup, j. Williams G.) Blackwell, London 1997, 2nd edn, vol. I, pp 1-21.

Moller, D.E., 2001. New drug targets for type 2 diabetes and the metabolic syndrome. Nature, 414: 821-827. PMID: 11742415

Mohammad Khalil, Gamal Mohamed, Mohammad Dallak, Fahaid Al-Hashem, Hussein Sakr, Refaat A. Eid, Mohamed A. Adly, Mahmoud Al-Khateeb, Saleh Banihani, Zuhair Hassan and Nabil Bashir (2010):The Effect of Citrullus colocynthis Pulp Extract on the Liver of Diabetic Rats a Light and Scanning Electron Microscopic Study. American Journal of Biochemistry and Biotechnology 6 (3): 155-163, 2010 ISSN 1553-3468

Naik SR, Barbosa Filho JM, Dhuley JN, Deshmukh V. "Probable mechanism of hypoglycemic activity of bassic acid, a natural product isolated from Bumelia sartorum". J. Ethnopharmacol. 1991; 33(1-2): 37-44.

Pushparaj PN, Tan BK, Tan $\mathrm{CH}$. "The mechanism of hypoglycemic action of the semipurified fractions of Averrhoa bilimbi in streptozotocin-diabetic rats". Life Sci. 2001; 70(5): 535-547.

Rajasekaran S, Sivagnanam K, Ravi K, Subramanian S. “Hypoglycemic Effect of Aloe vera Gel on Streptozotocin-Induced Diabetes in Experimental Rats". J. Med. Food. 2004; 7(1): 61-66.

Rollo J. “An account of two cases of the diabetes mellitus, with remarks as they arose during the progress of the cure". C. Dilly, London, 1797. 
Sanchez de Medina F, Gamez MJ, Jimenez I, Jimenez J, Osuna JI, Zarzuelo A.“'Hypoglycemic activity of juniper berries". Planta Med. 1994; 60(3): 197-200.

Sebbagh, N., C. Cruciani-Guglielmacci, F. Cruciani, Guglielmacci, M.F. Berthault and C. Rouch et al., 2009. Comparative effects of Citrullus colocynthis, sunflower and olive oil-enriched diet on streptozotocin-induced diabetes in rats. Diabetes Metab., 35: 178-184. PMID: 19264524

Seifalian, A.M., C. Piasecki, A. Agawel and B.R. Davidson, 1999. The effect of graded steatosis on flow in the hepatic on flow in the hepatic parenchymal microcirculation. Transplantation, 68: 780-784. PMID: 10515377

Sugihara Y, Nojima H, Matsuda H, Murakami T, Yoshikawa M, Kimura I."Antihyperglycemic effects of gymnemic acid IV, a compound derived fromGymnema sylvestre leaves in streptozotocin-diabetic mice". J. Asian Nat. Prod. Res. 2000; 2(4): 321-327.

Ugochukwu NH, Babady NE. "Antihyperglycemic effect of aqueous and ethanolic extracts of Gongronema latifolium leaves on glucose and glycogen metabolism in livers of normal and STZ-diabetic rats". Life Sci. 2003; 73(15): 19251938.

Ugochukwu NH, Babady NE. “Antioxidant effects of Gongronema latifolium in hepatocytes of rat models of non-insulin dependent diabetes mellitus". Fitoterapia 2002; 73: 612618.

Ugochukwu NH, Cobourne MK. "Modification of renal oxidative stress and lipid peroxidation in STZ-diabetic rats treated with extracts from Gongronema latifolium leaves". Clinica Chimica Acta 2003; 336: 73-81

Venkateswaran S, Pari L. "Effect of Coccinia indica leaves on antioxidant status in STZdiabetic rats". J. Ethnopharmacol. 2003; 84: 163-168.

Venkateswaran S, Pari L. "Effect of Coccinia indica on Blood Glucose, Insulin and Key Hepatic Enzymes in Experimental Diabetes". Int. J. Pharmacogn. 2002; 40(3): 165-170.

Waltner-Law, M.E., X.L. Wang, B.K. Law, R.K. Hall and M. Nawano et al., 2002. Epigallocatechin gallate, a constituent of green tea, represses hepatic glucose production. J. Biol. Chem., 277: 34933-34940. PMID: 12118006

WHO (1978a). Alma Ata Declaration. Primary Health Care. Health for all series No.1

World Health Organization, 1980. WHO expert committee on diabetes mellitus.

Witters L. "The blooming of the French lilac". J. Clin. Invest. 2001; 108: 1105-1107.

Yoshikawa M, Murakami T, Kadoya M, “Medicinal foodstuffs. III. Sugar beet. (1): Hypoglycemic oleanolic acid oligoglycosides,betavulgarosides I, II, III, and IV, from the root of Beta vulgaris L. (Chenopodiaceae)". Chem. Pharm. Bull. (Tokyo) 1996; 44: 1212-1217.

Yoshikawa M, Murakami T, Kadoya M, Li Y, Murakami N, Yamahara J, Matsuda H. "Medicinal foodstuffs. IX. The inhibitors of glucose absorption from the leaves of Gymnema sylvestre R. BR. (Asclepiadaceae): structures of gymnemosides a and b". Chem. Pharm. Bull. (Tokyo). 1997; 45(10): 1671-1676.

Yoshikawa M, Shimada H, Nishida N, Li Y, Toguchida I, Yamahara J, Matsuda H. "Antidiabetic principles of natural medicines. ALR2 and alpha-glucosidase 
inhibitors from Brazilian natural medicine, the leaves of Myrcia multiflora DC. (Myrtaceae): structures of myrciacitrins I and II and myrciaphenones A and B". Chem. Pharm. Bull. (Tokyo). 1998; 46(1): 113-119. 


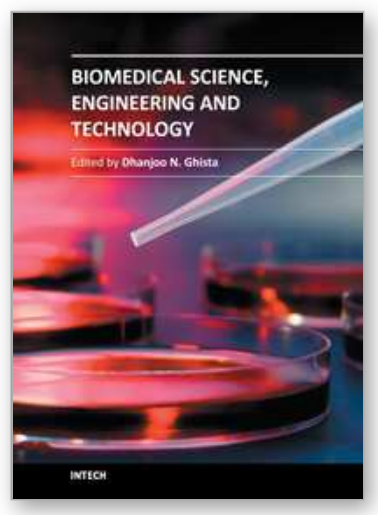

\author{
Biomedical Science, Engineering and Technology \\ Edited by Prof. Dhanjoo N. Ghista
}

ISBN 978-953-307-471-9

Hard cover, 902 pages

Publisher InTech

Published online 20, January, 2012

Published in print edition January, 2012

This innovative book integrates the disciplines of biomedical science, biomedical engineering, biotechnology, physiological engineering, and hospital management technology. Herein, Biomedical science covers topics on disease pathways, models and treatment mechanisms, and the roles of red palm oil and phytomedicinal plants in reducing HIV and diabetes complications by enhancing antioxidant activity. Biomedical engineering coves topics of biomaterials (biodegradable polymers and magnetic nanomaterials), coronary stents, contact lenses, modelling of flows through tubes of varying cross-section, heart rate variability analysis of diabetic neuropathy, and EEG analysis in brain function assessment. Biotechnology covers the topics of hydrophobic interaction chromatography, protein scaffolds engineering, liposomes for construction of vaccines, induced pluripotent stem cells to fix genetic diseases by regenerative approaches, polymeric drug conjugates for improving the efficacy of anticancer drugs, and genetic modification of animals for agricultural use. Physiological engineering deals with mathematical modelling of physiological (cardiac, lung ventilation, glucose regulation) systems and formulation of indices for medical assessment (such as cardiac contractility, lung disease status, and diabetes risk). Finally, Hospital management science and technology involves the application of both biomedical engineering and industrial engineering for cost-effective operation of a hospital.

\title{
How to reference
}

In order to correctly reference this scholarly work, feel free to copy and paste the following:

Ahmed Morsy Ahmed (2012). Medical Plant and Human Health, Biomedical Science, Engineering and Technology, Prof. Dhanjoo N. Ghista (Ed.), ISBN: 978-953-307-471-9, InTech, Available from: http://www.intechopen.com/books/biomedical-science-engineering-and-technology/medical-plant-and-humanhealth

\section{INTECH}

open science | open minds

\author{
InTech Europe \\ University Campus STeP Ri \\ Slavka Krautzeka 83/A \\ 51000 Rijeka, Croatia \\ Phone: +385 (51) 770447 \\ Fax: +385 (51) 686166 \\ www.intechopen.com
}

\author{
InTech China \\ Unit 405, Office Block, Hotel Equatorial Shanghai \\ No.65, Yan An Road (West), Shanghai, 200040, China \\ 中国上海市延安西路65号上海国际贵都大饭店办公楼 405 单元 \\ Phone: +86-21-62489820 \\ Fax: +86-21-62489821
}


(C) 2012 The Author(s). Licensee IntechOpen. This is an open access article distributed under the terms of the Creative Commons Attribution 3.0 License, which permits unrestricted use, distribution, and reproduction in any medium, provided the original work is properly cited. 